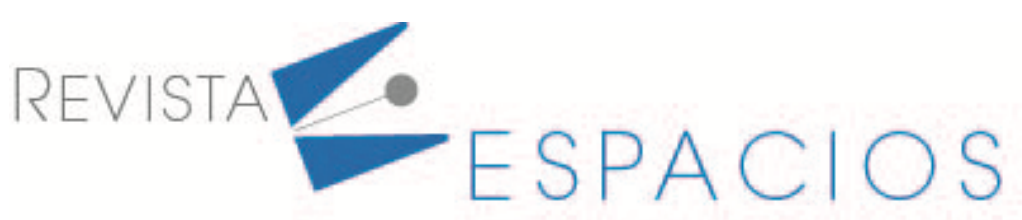

\title{
La filosofía como estigma de la actitud investigativa en educación superior
}

\section{Philosophy as a stigma of research attitude in higher education}

\author{
PACHECO Ruiz, Carlos Miguel ${ }^{1}$ \\ NIEBLES Nuñez, William Alejandro ${ }^{2}$ \\ HERNÁNDEZ Palma, Hugo Gaspar ${ }^{3}$
}

\begin{abstract}
Resumen
El artículo se orientó al análisis de la filosofía como limitación de la actitud investigativa en educación superior. Fue de tipo descriptivo de campo, no experimental. El muestreo fue estratificado contando con 166 estudiantes, la recolección de datos fue mediante la escala ACTINVES, con escala Likert. En el tratamiento estadístico se describieron los factores básicos y el análisis de varianzas. Se concluye que la filosofía realmente no estigmatiza la búsqueda del conocimiento y la orientación investigativa en procesos generales.
\end{abstract}

Palabras clave: filosofía, actitud, investigación, conocimiento

\begin{abstract}
The article focused on the analysis of philosophy as a limitation of the investigative attitude in higher education. It was descriptive type of field, not experimental. Sampling was stratified with 166 students, data collection was developed with ACTINVES scale, with Likert scale. The statistical treatment described the basic factors and analysis of variances. It is concluded that philosophy does not really stigmatize the pursuit of knowledge and research orientation in general processes.

key words: philosophy, attitude, research, knowledge
\end{abstract}

\section{Introducción}

La entrada de las nuevas tecnologías y su espacio dentro del entorno educativo ha marcado un antes y un después en los procesos de formación (Cárdenas, Redondo \& Palma, 2020), especialmente en las instituciones de educación superior, enfatizando en los procesos de investigación (Viloria et al, 2020) y mediación del conflicto (Pérez-Guardo, Tafur-Cabrera, Molina-Padilla y Hernández-Palma, 2019). La relación que existe entre la filosofía y la ciencia es indiscutible, las dos han sido fundamento para el desarrollo del saber humano transformándose

\footnotetext{
${ }^{1}$ Magister en Administración de Empresas, Especialista en Gerencia de Proyectos, Administrador de Empresas Docente Universidad de Sucre. carlos.pacheco@unisucre.edu.co.

2 Doctor en Ciencias Gerenciales, Magister en Dirección Estratégica, Administrador de Empresas. Docente de la Universidad de Sucre. ORCID: 0000-00019411-4583. Email: william.niebles@unisucre.edu.co

${ }_{3}^{3}$ Magister en Sistema de Gestión, Especialista en Estudios Pedagógicos, Especialista en Diseño y Evaluación de proyectos, Ingeniero Industrial. Docente Programa de Administración de Empresas Universidad del Atlántico- Colombia. Email: hugoghernandezpalma@gmail.com
} 
en las bases del conocimiento lógico y experimental gestándose a través del desarrollo de estas dos grandes fuentes de aprendizaje que han permitido la creación de todo un mundo de saberes a partir de los aportes y principios que de ellas se han derivado. Sin embargo, así como la filosofía y la ciencia se han servido una de la otra, también han encontrado sus enfrentamientos en la historia de su evolución y constitución; o más bien, el problema también radica en cuál de las dos fue primera, o en algunos casos quién termina sirviéndose de los avances que puede hacer la una por la otra.

Es así como en la confrontación en que se encuentran estas dos partes del ejercicio intelectual humano, se ha generado una situación de querella, pues académicos, científicos y todos los que aportan al avance de la ciencia y al conocimiento, han discutido la relación o distancia que hay entre estas. Desde la filosofía surgen elementos que promueven el desarrollo del saber y la ciencia complementa aspectos que requieren de una concepción filosófica. En este caso como se evidencian en la acción investigativa.

Aunado a ello, Moreno, et al (2018), han considerado que los elementos que generan acción filosófica se fundamentan en las creencias del hombre, dado que el existir de cada persona es la parte fundamental cuando el hombre actúa, y primordialmente en los momentos que este se guía por decisión propia, es en este punto donde se interrelaciona con su "existencia".

Se expresa un esbozo de las características de la filosofía y de la ciencia, con la finalidad de mirar en qué momento clave estos dos tipos de saberes encontraron sus diferencias, o si realmente una no se encontraba dentro de los parámetros de la otra. Atendiendo a las consideraciones anteriores, Hernandez et al (2018), enuncian que se ha determinado que la actividad del ser humano se ha caracterizado por presentar una diversidad de elementos estructurales, que a través del tiempo han demostrado diferentes maneras de construir de forma individual una posición conductual frente a alguna situación determinada.

La filosofía y la investigación que surge de la ciencia, son actividades humanas que producen un cúmulo de conceptos e ideas que han servido de pilares para el desarrollo científico y humanístico contemporáneo. Por su parte, la filosofía es necesaria tanto como lo es la ciencia, sin desdeñar el valioso aporte que cada una realiza, puesto que le brinda al hombre la oportunidad de encontrare a sí mismo.

Asimismo, el desarrollo de la actitud frecuentemente se tiende a singularizar con respecto a la manera como el individuo acoja un comportamiento determinado frente a algún elemento exterior que genera dichos cambios, aunado al proceso cognoscitivo que el individuo exteriorice.

En este sentido, Parra et al (2019), enuncian que cualquier formación de principios y conceptos de carácter conductual en el ser humano se reflejan al presentar situaciones de manera externa, los que propician respuestas accionares específicas y que conforman su personalidad. Dado esto, el proceso de enseñanza-aprendizaje en la gestión investigativa no está excluido de la formación de componentes que muestran comportamientos individuales. Dicho proceso evidencia, una vasta diversidad de factores que pudiesen producir comportamientos en el individuo, a partir de la educación de carácter generalizado impartida en instituciones educativas universitarias.

Por otro lado, las diversas unidades curriculares que se facilitan en estos espacios educativos pudiesen generar diversos comportamientos en los estudiantes basado en lo percibido. En este orden de ideas de concepción filosófica, la problemática está estructurada en un único marco, y además, se representa por las cátedras en el eje curricular "investigación", generando diversas modalidades de conducta en los estudiantes, permitiéndole tomar actitudes hacia la ejecución practica de la investigación y por consiguiente, a las inherentes actividades a la trama investigativa, lo cual regularmente condiciona los procesos destacando la problemática que genera la comprensión de los procesos filosóficos de la investigación, en relación a la epistemología y desarrollo del saber, como parte de la ciencia. 
En ese sentido, Rivera et al (2019), muestran que los estudiantes hoy en día manifiestan a través de sus negativas acciones hacia el proceso investigativo, en relación con las expresiones filosóficas, así como la sustentación de la episteme que debe conocerse para el accionar investigativo y los oriente al desarrollo del conocimiento.

Cabe destacar que uno de los problemas que se evidencian en la institución universitaria es que han surgido diversas unidades curriculares, cursos y seminarios, que evitan estructuralmente las realidades laborales y sociales de la nación, al desarrollar proyectos de investigación sin asociación con las necesidades investigativas (Steffens et al, 2017; Lay, Parra \& Ramírez, 2019).

Del mismo modo, ciertamente existe nulo interés por parte de los estados por desarrollar programas que impulsen la cultura investigativa tanto en docentes como en los estudiantes. Lo cual, pudiese relacionarse con la preparación deficiente del cuerpo docente acerca de la concepción filosófica que sustenta la praxis investigativa y que pueda promover una actitud integradora hacia el conocimiento.

En este sentido, Bauer, (2018), sostiene que la filosofía, como campo de estudio, ha impulsado grandes cambios en la humanidad, para el desarrollo obtenido en los tiempos modernos. Ha actuado en el cambio de regímenes a gobiernos. La corriente positivista, propuesta por la filosofía, permite un análisis de la humanidad en los campos histórico-filosóficos en su totalidad.

Para Mora, (2008), esta actúa como creencia con bases fundamentadas, es la motivación directa de la fundamentación de la ciencia. Las leyes, estimuladas inicialmente por las creencias, surgen como también como decisiones fundamentadas. La teoría funciona como ideal, aplicado en el servicio, de una comunidad y de una población para alinearla en el cumplimiento de ciertos objetivos, o principios base.

Siguiendo con lo estipulado anteriormente, se denota una situación alarmante acerca de las características expuestas por los alumnos cuando al participan en asignaturas como Metodología de la Investigación o semilleros de investigación, estos expresan supuestamente una aversión a dichas cátedras, causando altos índice de repitencia, considerando que uno de los aspectos que más influye son los contenidos filosóficos y epistemológico que sustenta el área de la investigación, y que en la realidad no satisfacen a los estudiantes.

Asimismo, se ha demostrado un alto índice de estudiantes repitientes, poco interés en la investigación y que además se les dificulta el aprendizaje de los contenidos y secuencias operativas aplicadas en dichas asignaturas, posiblemente causado por la formación de las cátedras básicas, la cual al vincularse con las aptitudes del desarrollo del pensamiento, en donde las herramientas de lectura, análisis e interpretación, no han sido eficientes; del mismo modo se muestran debilidades al argumentar, sintetizar y analizar los contenidos. En virtud de lo planteado, los investigadores se orientaron a indagar como la filosofía se concibe como un estigma de la actitud investigativa en educación superior.

\section{Metodología}

El presente estudio se considera de tipo descriptivo de campo. Según Méndez (2012), este tipo de investigaciones se efectúan en función de identificar los componentes que intervienen en la problemática; o, dicho de otra forma, estipulando las posibles situaciones vinculadas en el establecimiento del comportamiento del alumnado hacia la investigación. La investigación de campo se desenvolvió a partir del entendimiento de la realidad, de los contenidos y de los elementos que interceden en el contexto educativo sin el establecimiento de ningún control.

Por otra parte, el diseño fue no experimental descriptivo, lo cual definen Hernández, Fernández y Baptista (2014), como los estudios que propician el establecimiento de comportamiento y perspectiva general de alguna variable en conjuntos de personas en un momento específico, sin la manipulación deliberada de la variable. 
Para ejecutar la investigación, se usó un muestreo estratificado el cual contó con 166 estudiantes, quienes se les distribuyó en 8 secciones donde se les imparte Metodología de Investigación I y II. Para recolectar los datos se efectuó a través de la escala ACTINVES, usando los criterios de medida tipo Likert. Dicho instrumento se encuentra integrado de la siguiente manera:

Tabla 1

Distribución del instrumento

\begin{tabular}{|c|c|c|}
\hline Dimensión & Subdimensión & indicador \\
\hline \multirow{4}{*}{$\begin{array}{l}\text { Componente } \\
\text { Filosófico }\end{array}$} & \multirow[t]{2}{*}{ Emociones hacia la filosofía } & Disposición \\
\hline & & Emociones hacia el docente \\
\hline & \multirow[t]{2}{*}{ Sentimientos sobre la filosofía } & Búsqueda del desarrollo del saber \\
\hline & & Sentimientos hacia el docente \\
\hline \multirow{4}{*}{$\begin{array}{l}\text { Componente } \\
\text { cognitivo }\end{array}$} & \multirow[t]{2}{*}{ Juicios sobre la investigación } & Juicios establecidos sobre la asignatura \\
\hline & & $\begin{array}{l}\text { Juicios establecidos sobre la } \\
\text { investigación }\end{array}$ \\
\hline & \multirow{2}{*}{$\begin{array}{l}\text { Creencias Sobre la } \\
\text { investigación }\end{array}$} & Juicios establecidos sobre la asignatura \\
\hline & & $\begin{array}{c}\text { Juicios establecidos sobre la } \\
\text { investigación }\end{array}$ \\
\hline \multirow[t]{3}{*}{$\begin{array}{l}\text { Componente } \\
\text { conductual }\end{array}$} & $\begin{array}{l}\text { Intenciones hacia la } \\
\text { investigación }\end{array}$ & $\begin{array}{l}\text { Favorecimiento a las unidades } \\
\text { curriculares }\end{array}$ \\
\hline & \multirow[t]{2}{*}{$\begin{array}{l}\text { Tendencias Hacia la } \\
\text { investigación }\end{array}$} & $\begin{array}{l}\text { Imita lo establecido en grupos de } \\
\text { referencia }\end{array}$ \\
\hline & & Participación \\
\hline
\end{tabular}

Fuente: Elaboración propia (2020)

En este sentido, se explica que en el tratamiento estadístico se describieron los factores básicos y el análisis de varianzas y fue realizado en el software estadístico SPSS.

\section{Resultados}

\subsection{Identificación de los elementos del comportamiento en los estudiantes}

Utilizar la estadística descriptiva concedió la expresión de los elementos generales de esta, basados en tres aspectos los que constituyen la medición de la actitud: afectivo, cognitivo y conductual. Siguiendo la información de la tabla que se observan a continuación, sobre la aplicación de la escala ACTINVES, la puntuación media establecida por el total de la población fue de 2,73, siendo esta una tendencia de medida central, y entre los valores de 1 y 4.

SE obtuvo una mediana 2,82 y la moda reflejó la opción 2, lo cual es representación de un factor con irregularidad respecto a los resultados conseguidos en los componentes individualmente. En la muestra, la desviación estándar se estableció en 4,7 lo cual indica que la data se encuentra dispersa de manera moderada con respecto a al promedio. 
Tabla 2

Componentes de la actitud investigativa

\begin{tabular}{|c|c|c|c|c|}
\hline $\begin{array}{c}\text { Estadística } \\
\text { descriptiva }\end{array}$ & $\begin{array}{c}\text { Compt } \\
\text { Filosófico }\end{array}$ & $\begin{array}{c}\text { Compot } \\
\text { conductual }\end{array}$ & $\begin{array}{c}\text { Compt } \\
\text { cognitivo }\end{array}$ & Total \\
\hline N & 166 & 166 & 166 & 166 \\
Media & 2,90 & 2,83 & 2.61 & 2,73 \\
Mediana & 2,90 & 3,00 & 2,62 & 2,82 \\
Moda & 4 & 4 & 3 & 2 \\
Desviación E &, 92 &, 91 &, 51 &, 52 \\
Varianza &, 87 &, 89 &, 19 &, 19 \\
Asimetría &,- 274 &,- 270 &, 463 &,- 260 \\
Curtosis & $-1,023$ & $-1,097$ &, 537 &,- 169 \\
Mínimo & 1 & 1 & 1 & 2 \\
Máximo & 4 & 4 & 4 & 4 \\
Percentil 25 & 2,00 & 2,00 & 2,30 & 2,38 \\
Percentil 50 & 3,00 & 3,00 & 2,70 & 2,77 \\
Percentil 75 & 2,67 & 2,67 & 3,00 & 3,10 \\
\hline
\end{tabular}

Fuente: Elaboración propia (2020)

Con respecto a las medidas de dispersión, la varianza que se encuentra en 0,19, se evidencia una variación en la escala por encima o debajo de la data de la población. En cuanto al análisis de la curva, se obtuvo un indicador de asimetría negativo -0,260, mostrando la posición de los valores se encuentran ubicados un poco por debajo de la media. Al estudiar la curtosis se refleja que esta es de -0,169, en los resultados de la prueba, estableciendo una curva dispuesta en poca medida, a condiciones de alineación normal (platicurtica), puesto que el valor es de menos de 3.

Sobre las medidas de orden que resultaron de aplicar la escala ACTINVES, se contempló, como expone la Tabla 3 que $30 \%$ de las puntuaciones es igual o por debajo de 2,0 de los sujetos de estudio, correspondiendo a una escasa variación relativa. Asimismo, $64 \%$ en las puntuaciones fueron menores o iguales a 3 y, el último $6 \%$ sobrepaso la puntuación establecida (entre 4 y 5), o, dicho de otra manera, alrededor de 8 de los participantes tuvieron puntuaciones mayores a 4.

Tabla 3

Medidas de orden

\begin{tabular}{cc}
\hline $\begin{array}{c}\text { Alternativas de la } \\
\text { Escala }\end{array}$ & $\begin{array}{c}\text { Porcentaje acumulado } \\
\text { Total }\end{array}$ \\
\hline$<2$ & $30 \%$ \\
3 & $60 \%$ \\
Entre 4 y 5 & $6 \%$ \\
\hline Fuente: Elaboración propia (2020)
\end{tabular}

Lo evidenciado anteriormente, permite la significativa consideración de las medidas de orden de carácter individual en todos los elementos de la variable en estudio; por lo cual se posibilita su establecimiento según lo contemplado en relación con el factor filosófico, $94 \%$ se representa con una puntuación menor o igual a 3, lo cual manifiesta la variabilidad entre la actitud favorable para con la investigación y la neutralidad de la contestación.

Asimismo, las favorables disposiciones de los participantes a la práctica de la investigación interpretada por un factor de concepción filosófica, donde la expresión del conocimiento viene por las estimulaciones externas recibidas, lo cual lleva al establecimiento de demostraciones de exaltación a los contenidos impartidos en las clases de Metodología de Investigación I y II, y especialmente que la percepción de los educadores es positiva, acerca de impartir dichas cátedras. 
Desde esa perspectiva, la significativa aceptación que los estudiantes presentan respecto al elemento filosófico es organizado de manera consecutiva para lograr el estímulo que éstos perciben para realizar investigaciones con vinculación metodológica, disposición y satisfacción por las lecciones y estímulo para ejecutar investigaciones. Las bases filosóficas que consideran importante los estudiantes evidencian equilibrios favorables entre aquellos aspectos analizados.

Sobre el elemento cognitivo, $90 \%$ de las puntuaciones que se obtuvieron de lo respondido por los participantes, se encuentra ubicada en el rango de menor o igual a 3, significativamente equitativo al filosófico, evidenciando creencias neutrales a la práctica en investigación, reflejando tendencias propicias a las acciones propias al quehacer de la investigación.

En Relación a comprensión y conceptualización en el fundamento conceptual o teórico de las asignaturas que componen la investigación, logra evidenciarse que una porción de los participantes objetos de estudio expresó una favorable disposición, en función de la educación y percepción; permitiendo la identificación de congruencias referenciadas entre la educación de los contenidos del programa y la información recibida por los alumnos.

Consecuentemente, las premisas que terminan reflejando este elemento organizan el pensamiento y conocimiento del alumno para que se considere la Investigación como un eje curricular fundamental en los programas académicos de las instituciones de educación superior públicas de las Costa Caribe. Normalmente, lo simple para entender la unidad educativa y la secuencia operativa que se ha desarrollado en los proyectos investigativos de estos, convierte en significativas y llamativas las cátedras que permean este eje.

Siguiendo el esquema de análisis, la información obtenida, en lo que respecta al componente de la actitud (Conducta), tomaron un matiz de parámetros similares en este estudio. Un $70 \%$ de los datos recolectados se ubican parecidamente hasta 3,0 (incluido). Se puede inferir, que la conexión en los procesos mediadores con la respuesta a los estímulos, respuesta hipotéticamente señalada en individuos, reafirman posiciones favorables que coadyuvan a mejorar las acciones en el desarrollo de la investigación, tal es el caso, del resultado de la actitud que los estudiantes asumen ante las propuestas del currículo en asignaturas de metodología de investigación y metodología de investigación II, evidencian cierta los efectos en los alumnos en su comportamiento o actitud frente a distintas situaciones que enfrentan o en la praxis de la investigación.

Ahora, la generación de actitudes en estudiantes depende de la realización de actividades curriculares, asumir conductas homogéneas en los grupos, de la incidencia del grupo y la las relaciones interpersonales que persuadan a otros a asumir la conducta deseada. En cuanto a considerar las asignaturas relacionadas con la investigación como un medio adecuado para generar y divulgar el conocimiento, se ve condicionado a la experiencia que adquiera y acumule el estudiante, también por las palabras o expresiones que su grupo de referencia haga al respecto

En concordancia con el análisis de la data, Villareal et al (2019), evidencia una orientación de la actitud en lo que concierne a asignaturas afines a la investigación como una experiencia cognitiva del individuo que favorece su relacionamiento con el entorno, debido que existe una conducta y afinidad emotiva en casi todas las particularidades del contexto.

De acuerdo con lo expresado, se destaca que la filosofía como fundamento de los contenidos curriculares, es más que una ciencia, Millán, (2001), considera que es la forma más perfecta, que se ocupa de todo ente y, por tanto, según su más profundo y entrañable sentido. Bunge (2014), este propone que la actividad científica pertenece al ámbito social de la vida; justo cuando esta es aplicada en función de mejorar el medio artificial y natural, a la inventiva y producción de bienes de carácter material y cultural, dividiendo las ciencias en dos vertientes fácticas (o materiales) y formales (o ideales). 
Esta clasificación da entender que las ciencias tienen dos grandes ramas: las que su objeto de estudio son entes formales, como las matemáticas que construyen a través de un ejercicio lógico y de abstracción todos sus axiomas, principios y teorías y cuyo resultado se genera a través de un proceso de deducción donde se elaboran sus postulados y teoremas sin necesidad de ir a la experimentación; y las ciencias fácticas que su objeto de estudio es material, basándose en la experimentación, dado como se relaciona con la actitud investigativa y más aún en relación a los nuevos procesos tecnológicos (Cárdenas, Redondo \& Palma, 2020).

Martínez, et al (2018), manifiestan que estos elementos parten de la educación, que requiere de la implementación de actividades tecnológicas y creativas; las cuales permiten a los estudiantes conocer, aprender, y adiestrarse en utilizar este tipo de instrumentación, de manera que se posibilite el alcance de dos objetivos: asimilar los conocimientos necesarios para su educación y actualizar el uso de las innovaciones.

A este respecto, se evidencian las etapas de la construcción del conocimiento.

Figura 1

Etapas de construcción del método científico

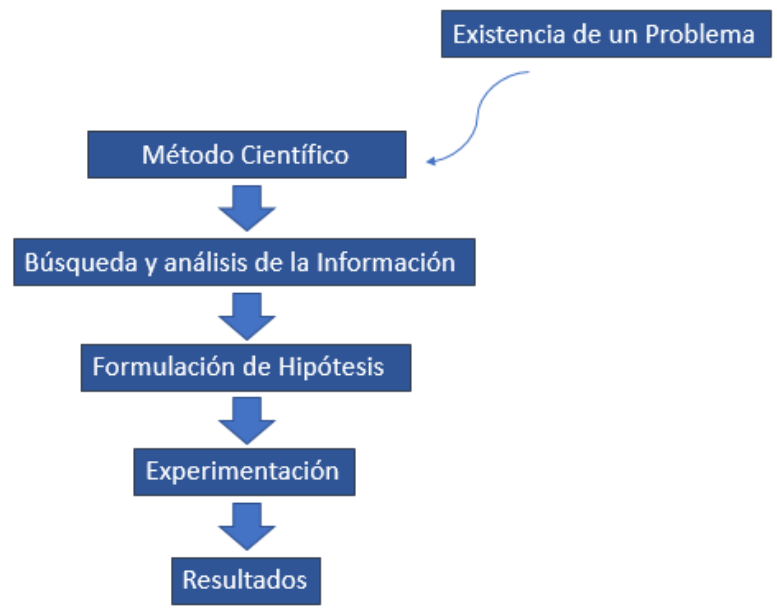

Fuente: Elaboración propia (2020)

De acuerdo a la figura 1, las etapas fundamentales para la construcción del método científico se orientan a la determinación de un problema: algo que requiere ser estudiado y no tiene inicialmente planteada una solución; recolección de la información: a través de la investigación de información de diferentes fuentes y/o bases de datos relacionados al problema; hipótesis: explicaciones planteadas como solución del problema estudiado; comprobación de hipótesis: procedimientos, experimentos destinados a la verificación de la certeza y validez de la información fundamentada en la etapa de la hipótesis.

Sin embargo aunque la esencia de la filosofía es ser discursiva, no teniendo la obligación de comprobar experimentalmente lo que afirma con un método específico, sino haciéndolo de diversas maneras y que en la variedad de aportes y perspectivas no se llegue a una verdad absoluta, esta le aporta un elemento relevante a la actitud investigativa.

Dado que la concepción filosófica, busca cuestionar lo que se le presenta como inquietante, dicho de otro modo, no se le pide dar soluciones a los problemas o interrogantes que se dan en la realidad, sino dar respuestas que no necesariamente deber ser tenidas como fijas, lo que la convierte en un saber enriquecedor, y es lo que se quiere impulsar en las actitudes investigativas en el estudiante.

La filosofia tiene relacion indudable con la promocion del desarrollo cognitivo, en opinion Mundó y Raventós, (2000), es dependiente de los acontecimientos a nivel social, de la evolución cultural y de otras características 
que son estudiadas por el estudio de la sociedad y de la comunidad. El desarrollo social, es la realidad que enfrenta el ser humano con sus emociones y hechos de supervivencia.

Dentro de estas perspectivas, la generación de nuevas ideas, planteamientos y conocimientos del ser humano lo cual según Garcia et al (2018), debe ser aprovechada para el uso de nuevas tecnologías que se imponen en el mercado, para Vilches y Gil, (2015), som aquellas denominadas limpias y verdes, que promueven el cuidado de la salud pública y privada de toda una nación, de los recursos de la naturaleza, y que incentiven en los nuevos gobiernos leyes y normativas de cuidado al medio

Cabe destacar que la filosofía surge en la generación de ideas en la necesidad de explicar todo el universo, relacionándose el hombre, el universo, y todos los medios para esclarecimiento y explicación del objeto de estudio, lo que induce a que el estudiante pueda lograr a traves del desarrollo del conocimiento, su actitud hacia la invetsigacion.

\section{Conclusiones}

La investigación y el análisis objetivo de la realidad favorecen la obtención de encontrar soluciones factibles y seguros a problemas objeto de un análisis. El método científico, de conformidad con los referentes teóricos y la lógica del investigador permite resolver una problemática, caso de estudio, generar nuevos procesos tecnológicos o comprender fenómenos (De la Hoz, Martínez, Combita \& Hernández, 2019).

Lo expuesto anteriormente es el inicio del desarrollo del presente estudio, entender desde las bases científica los aspectos que consienten la actitud, los resultados encontrados evidencian las consideraciones lógicas suficientes para disponer de valoraciones generales en cuanto a la conducta relacionadas con la investigación en los estudiantes que formaron parte de la muestra de estudio. El contexto descrito, permitió saber cómo influye la filosofía en los comportamientos encontrados en los estudiantes cuando llevan a la practicas los conceptos desarrollados en los currículos de investigación. Igualmente, se pudo enmarcar con presupuestos lógicos y científicos los aspectos o componentes de la actitud durante el proceso de investigar.

A continuación, se detalla organizadamente las condiciones del análisis descriptivo:

Componente filosófico: se caracteriza porque la globalización de los hallazgos, reveló la disposición normal de los estudiantes hacia el desarrollo practico de la investigación con la posibilidad de generar afinidad, desde la base de su disposición del conocimiento científico, buscar el desarrollo del saber y su percepción hacia el docente facilitador de los preceptos de acción investigativa.

Componente cognitivo: se caracterizó porque la correlación conceptual y proceso cognoscente, se orienta a la mediatización favorable.

Componente conductual: se caracteriza en cuanto a que la actuación mostrada entre los sujetos fue armónica; evidenciándose una neutralidad conveniente en los currículos a fines a la investigación conforme al resto de los componentes. Esta actitud se manifestó en el desarrollo de las actividades propuestas de dichas asignaturas y de la homogeneidad de comportamiento respecto a los grupos de referencia

Esta evidencia, devela que el comportamiento presentado por los estudiantes es importante para: definir homogeneidad en el diseño de los currículos en investigación formativa, en las competencias de los docentes, las formas de transmitir conocimiento y la capitalización de experiencias fruto del aprendizaje, favoreciendo una actitud orientada al análisis investigativo.

El establecimiento de la actitud en los estudiantes desde el diseño de currículos en asignaturas a fines a Metodología de la Investigación, es fundamental para que los estudiantes muestren objetivamente el análisis 
que realizan para resolver situaciones concretas basadas en su aprendizaje, la cual se origina desde las concepciones filosóficas que hace un triada del saber, el hacer y el conocer.

Por consiguiente, la filosofía favorece la comprensión de los fenómenos a estudiar y seguidamente avala la solución por medio de la calidad del método escogido, debido que confirma que el entendimiento y la capacidad de pensamiento son propicios cuando se desea resolver procesos complejos. Por lo tanto, de ninguna manera la filosofía no estigmatiza la obtención del conocimiento ni tampoco la perspectiva de la investigación en procesos generalizado.

\section{Referencias bibliográficas}

Bauer, C. (2018). Las marcas del positivismo en la historia y la filosofía latinoamericanas. Filosofia \& Educação. 10(3), 506-529.

Bunge, M. (2014). La ciencia, su método y su filosofía. Sudamericana.

Cadenas, M. J., Redondo, R. P., \& Palma, H. H. (2020). Las Tecnologías de la Información y las Comunicaciones para la promoción de La educación en Colombia. Prospectiva, 18(2).

De la Hoz, E., Martínez, O., Combita, H., \& Hernández, H. (2019). Las Tecnologías de la Información y la Comunicación y su Influencia en la Transformación de la Educación Superior en Colombia para Impulso de la Economía Global. Información tecnológica, 30(1), 255-262.

Gadamer, H. (2001). Subjetividad, Sujeto y Persona. Tomado de: El giro hermenéutico. Madrid: Ediciones Cátedra.

Hernandez, I., Parra M., García, J., Castro N., Romero C., \& Duran. S. (2018). Comportamiento Organizacional Ciudadano (COC) como ejemplo de participación generador de un diálogo de saberes, Revista Espacios, Volumen 39, numero, 7 Caracas Venezuela. Recuperado, marzo 08 de 2018. En http://www.revistaespacios.com/a18v39n07/18390722.html

Hernández, R., Fernández, C., \& Baptista, P. (2014). Metodología de la investigación. Sexta Edición. Editorial Mc Graw Hill. México. 2014• Hernández, R. Metodología de la Investigación. 6a Edición, Mc Graw Hill, México.

Lay, N., Parra, M., \& Ramírez, J. (2019). Desarrollo de conductas ciudadanas en estudiantes del octavo grado de una institución educativa de Barranquilla. I Congreso Internacional en Educación e Innovación en Educación Superior. Caracas: Universidad Pedagógica Experimental Libertador.

Martínez, O., Steffens, E. J., Ojeda, D. C. Y Hernández, H. G. (2018). Estrategias pedagógicas aplicadas a la educación con mediación virtual para la generación del conocimiento global. Formación universitaria, 11(5), 11-18.

Millán-Puelles, A. (2001). Fundamentos de filosofía (Vol. 3). Ediciones Rialp.

Mora, A. (2008). Filosofía y ciencia. Rev. Filosofía Univ. Costa Rica, 69-74.

Moreno, M., Tezón, M., Rivera, T., Durán, S., \& Parra, M. (2018). Autoestima: Desarrollo de la autonomía personal en estudiantes del área técnica. Revista Espacios, 39, 46. En http://www.revistaespacios.com/a18v39n07/18390722.html

Mundó, J., Raventós, D. (2000). Fundamentos Cognitivo-Evolucionarios de las Ciencias Sociales. Revista Internacional de Sociología. 58(25), 1-28. 
Parra, M. Visbal, O. Duran, S. Badde, G. Calidad de la comunicación y actitud de los empleados ante procesos de cambio organizacional Interdisciplinaria, doi: https://doi.org/10.16888/interd.2019.36.1.11, Centro Interamericano de Investigaciones Psicológicas y Ciencias Afines, 36 (1), 155-170 (2019)

Pérez-Guardo, C., Tafur-Cabrera, J., Molina-Padilla, G., \& Hernández-Palma, H. (2019). Análisis del Papel del Educador en el Postconflicto. Un Acercamiento desde la Percepción de la Ciudad de Barranquilla, en Colombia. Información tecnológica, 30(4), 199-208.

Rivera, J., Lay, N., Moreno M. E., Pérez, A., Rocha, G., Parra, M, Duran S, Torres E., ... \& Redondo O. (2019) Programa de entrenamiento para desarrollar habilidades sociales en estudiantes universitarios. Revista espacios. Volumen 40 numero 31. Páginas 10-25.

Steffens, E., J Ojeda, D. D. C., Martínez, O. M., García, J. E., Hernández, H. G., \& Marin, F. V. (2017). Niveles de pensamiento crítico en estudiantes de Universidades en Barranquilla (Colombia). Revista Espacios, 38(30).

Vilches, A., \& Gil, D. (2015). Ciencia de la Sostenibilidad: ¿Una nueva disciplina o un nuevo enfoque para todas las disciplinas? Revista Iberoamericana de Educación. 69(1), 39-60.

Villarreal, S. García, J. Hernández, H. \& Steffens, E. (2019). Competencias Docentes y Transformaciones en la Educación en la Era Digital. Formación universitaria, 12(6), 3-14. Doi: https://dx.doi.org/10.4067/S071850062019000600003

Viloria, A., Naveda, A. S., Palma, H. H., Núñez, W. N., \& Núñez, L. N. (2020). Using Big Data to Determine Potential Dropouts in Higher Education. En Journal of Physics: Conference Series. IOP Publishing.

Esta obra está bajo una Licencia Creative Commons Attribución-NoCommercial 4.0 International

\section{(cc) EY-NC}

